\title{
CONCEPTS
}

\section{Participation of Iatrogenically Coagulopathic Patients in Wilderness Activities}

\author{
Seth C. Hawkins, MD; Michael J. Caudell, MD; Thomas G. Deloughery, MD; William Murray, BA \\ From the Department of Emergency Medicine, UNC-Chapel Hill School of Medicine, Chapel Hill, NC (Dr Hawkins); Department of \\ Emergency Medicine, Georgia Health Sciences University, Augusta, GA (Dr Caudell), Division of Hematology-Oncology, Oregon Health \& \\ Science University, Portland, OR (Dr Deloughery), and the NC Outward Bound School, Asheville, NC (Mr Murray).
}

\begin{abstract}
An increasing number of patients routinely undergo long-term anticoagulation with warfarin or other pharmacological agents. There is little evidence and no consensus documents in the literature regarding the appropriateness and relative risk of their participation in wilderness activities. We present a case report, conduct an analysis of the limited literature that is available, and make recommendations for wilderness medicine practitioners and screening personnel.
\end{abstract}

Key words: coagulopathy, warfarin, wilderness activities, anticoagulation, outdoor sports, outdoor activities

\section{Introduction}

Anticoagulation is defined as "the process of hindering the clotting of blood," and coagulopathy is "a disease or condition affecting the blood's ability to coagulate [clot]." "The Greek roots to the term iatrogenic literally mean "a condition caused by a physician," whether that result was intended or not. In the case of iatrogenic coagulopathy, anticoagulation is intentional, with the goal of reducing the blood's ability to clot to prevent another complication that is presumably higher risk. Examples in medicine when iatrogenic coagulopathy is used include conditions in which clotting must be prevented or is at a higher risk of occurring, such as in patients with artificial valves, chronic, clot-prone arrhythmias such as atrial fibrillation, or a history of embolic strokes (strokes caused by clots). Because this intervention is intentionally disrupting a normal function of the body (clotting), bleeding risks are higher in these patients; therefore, the risks and benefits of this intervention must be carefully and continually weighed by the caregiver and patient.

An increasing number of patients are routinely anticoagulated for long periods, including anticoagulation at younger ages. Long-term anticoagulation is typically

Corresponding author: Seth C. Hawkins, MD, Department of Emergency Medicine, UNC-Chapel Hill School of Medicine, 2201 South Sterling Street, Chapel Hill, NC 28655-0012 (e-mail: hawk@aya.yale. edu). achieved with warfarin (Coumadin, Jantoven). Recently, other oral anticoagulant agents have been approved, including apixaban (Eliquis), rivaroxaban (Xarelto), and dabigatran (Pradaxa), with slightly different pharmacological pathways from warfarin. ${ }^{2}$ In addition, there is some thought that agents that inhibit clotting via impairment of platelet activity, such as aspirin (Ecotrin, Bayer, St. Joseph) and clopidogrel (Plavix), should be considered in the same risk pool as other more traditional anticoagulants like warfarin. Although there are a substantial number of studies evaluating the relative risk of anticoagulated patients in trauma situations, it is not clear which subsets, if any, of these data can be directly translated into wilderness medical environments. There are also no consensus documents or even case reviews available in the literature addressing the appropriateness and relative risk of patients with long-term anticoagulation participating in wilderness activities.

We present a recent clinical case that prompted a review of the risks for patients on warfarin in the wilderness. We discuss the findings of our literature search and the evidence used in making decisions for the case patient. We also discuss not only how the available evidence may be helpful for making decisions in the wilderness operational environment but also important ways in which the available evidencelargely from the trauma, emergency medicine, radiology, and neurosurgery literature-may not be appli- 
cable to the wilderness operational environment. Finally, we provide general recommendations for physicians advising or screening anticoagulated individuals who want to participate in wilderness activities and for management of anticoagulated patients sustaining traumatic injury in the wilderness.

\section{Case Study}

Outward Bound, which marks its 50th year of operations in the United States in 2012, is one of the oldest and most well-known wilderness experiential education schools in the country. ${ }^{3}$ Outward Bound has a number of charter schools, among them the North Carolina Outward Bound School (NCOBS).

In September 2011, a 42-year-old man with a history of deep venous thrombosis (DVT) applied to an NCOBS program involving top-rope rock climbing and backcountry hiking with a 50-plus-pound backpack. The patient's DVT history was being managed via long-term use of warfarin, and his private physician confirmed that his international normalized ratio (INR), a laboratory value used to measure warfarin's effect, had been within therapeutic ranges in the months before the application. The NCOBS program involved 5 days in the Pisgah National Forest near the Linville Gorge Wilderness Area. The participants would never be more than 1 mile from a Forest Service road. The patient had no significant prior rock-climbing experience.

The patient's application was declined owing to current Outward Bound guidelines that anticoagulated patients could not be taken into the backcountry. The patient and his private physician appealed this decision, stating that they felt the activities were well within the safe range of activities for an iatrogenically coagulopathic patient. The NCOBS consulted their physician medical advisor.

\section{Review of Warfarin Usage and Pharmacology}

Warfarin's antithrombotic mechanism acts via interference of vitamin K-dependent gamma-carboxylation of coagulation proteins II, VII, IX, and X. As a result these coagulation factors cannot bind calcium. This causes both hindering of formation of protein secondary structures and inability of these proteins to bind membranes, resulting in less effective coagulation. The INR measures the anticoagulant effect of warfarin. A range of 2 to 3 is used for most indications, but for certain mechanical prosthetic cardiac valves higher ranges are used. ${ }^{4}$

Unfortunately, the dose of warfarin required for achieving therapeutic anticoagulation varies among patients. Dosing can range from $1 \mathrm{mg}$ daily to as high as 40 mg daily. This is because of a combination of the patient's genetic ability to metabolize warfarin, concurrent medications, and diet. This variability necessitates frequent monitoring of patients' INRs to make certain they are in therapeutic range. Experts recommend that, even for stable patients, an INR be checked once a month, and that caregivers check the INR more often if levels are unstable. ${ }^{5}$

Given the complex metabolism of warfarin, many medications can have an effect on dosing. Most interactions occur via agents metabolized by the enzyme cytochrome $\mathrm{P} 450$, family 2 , subfamily $\mathrm{C}$, polypeptide 9 (CYP2C9) and drugs that inhibit its action. These interactions augment the effect of warfarin. For example, trimethoprim-sulfamethizole inhibits CYP2C9, so a patient on warfarin who starts this agent will have an increase in their INR during the next few days unless the warfarin dose is reduced. A list of agents that increase warfarin's effect is included in Table 1.

Daily intake of vitamin $\mathrm{K}$ will also alter the effects of warfarin and therefore the warfarin dose. ${ }^{6}$ Patients who eat diets rich in vitamin $\mathrm{K}$ will need a higher dose of warfarin, whereas those who ingest minimal amounts will need smaller doses. Table 2 lists foods rich in vitamin K. Reduced intake of vitamin K can also lead to an unstable INR. Warfarin users will often avoid any vegetables because of fear of reversing their anticoagulation. This may result in those patients having lower vitamin $\mathrm{K}$ stores, and will make them prone to unstable INRs. Therefore, consistency in diet-especially vitamin $\mathrm{K}$ intake-is more important than avoiding vitamin $\mathrm{K}$ for maintaining a stable INR. ${ }^{7}$ In the wilderness setting this can be a challenge. For trips lasting more than a few days, dietary sources of vitamin $\mathrm{K}$ may be taken to maintain steady vitamin $\mathrm{K}$ intake (Table 2 indicates foods with high dietary sources of vitamin $\mathrm{K}$ ).

Several factors specific to wilderness activities can affect the INR. Patients with febrile illness will often have an increase in the INR, and this can be augmented if there is lack of oral intake or profuse diarrhea. Although poorly studied, it has been the observation of some anticoagulation clinics that patients who suddenly increase their activity level will have higher warfarin requirements. Data on altitude are also limited, but the same increase in warfarin requirements is also seen in patients who go to altitude. ${ }^{8}$ Of course warfarin may not be the only anticoagulation agent patients are taking. Many patients use antiplatelet agents such as aspirin or clopidogrel. These would be expected to increase bleeding from minor wounds or lacerations as a result of their antiplatelet effects. With the combination of aspirin and clopidogrel there is potential for severe bleeding equivalent to that of warfarin. ${ }^{9}$ Recently, novel oral anticoag- 
TABLE 1. Medication effects on warfarin effect

\section{Increased warfarin effect}

Acetaminophen

Alcohol (if concomitant liver disease)

Amiodarone

Anabolic steroids ${ }^{a}$

Azithromycin

Aspirin $^{a}$

Bismuth subsalicylate

Cephalosporins (NMTT group ${ }^{b}$ )

Cimetidine $^{a}$

Ciprofloxacin

Diltiazem

Erythromycin $^{a}$

Fluconazole $^{a}$

Furosemide

Isoniazid

Itraconazole $^{a}$

Ketoconazole $^{a}$

Metronidazole $^{a}$

Micanozole oral gel and vaginal suppositories

Omeprazole

Phenylbutazone

Propranolol

Quinidine $^{a}$

Quinine $^{a}$

Quinolones

Serotonin reuptake inhibitors

Sertraline

Sulfinpyrazone $e^{a}$

Tetracycline $^{a}$

Tricyclic antidepressants

Vitamin $\mathrm{E}^{a}$

Decreased warfarin effect

Barbiturates $^{a}$

Carbamazepine

Chlordiazepoxide

Cholestyramine

Corticosteroids

Etodolac

Griseofulvin

Mercaptopurine

Nafcillin

Phenytoin (may potentiate warfarin at initiation of drug)

Ribavirin

Rifampin

Sucralfate

Trazodone

Vitamin K

\footnotetext{
${ }^{a}$ Major effect.

${ }^{b}$ Cephalosporins which include a NMTT ( $N$-methylthiotetrazole) side-group; examples include cefmetazole and cefotetan.

Table 1 data were derived from DeLoughery, ${ }^{10}$ Nutescu et al., ${ }^{11}$ White, ${ }^{12}$ Bungard et al., ${ }^{13}$ and Holbrook et al. ${ }^{14}$
}

ulants that block either factor Xa (apixaban and rivaroxaban) or thrombin/factor IIa (dabigatran) have been studied in multiple phase III trials and are being approved for use by regulatory authorities. ${ }^{15}$ The new agents have no need for monitoring, lack food or drug interactions, and have a similar risk of bleeding compared with warfarin. Unlike warfarin and heparin, there are no standard antidotes for the new anticoagulants. For dabigatran use of prothrombin complex concentrates or activated prothrombin complex concentrates has been suggested at a dosage of 50 units $/ \mathrm{kg}$. For the factor Xa inhibitors such as rivaroxaban or apxiaban prothrombin complex concentrates are also recommend at the same dosing. There is also in development a novel "universal" antidote to reversal all $\mathrm{Xa}$ inhibitors which involves using recombinant factor $\mathrm{Xa}$ that has been rendered inactive by removal of the gla-domain and an active site modification. ${ }^{16}$

\section{Review of Outward Bound Policy on Anticoagulated Patients}

The NCOBS, in conformance with Outward Bound, Inc.'s National Safety Policies \& Standards, uses a welldeveloped medical screening process. This process includes both self-report and physician report components to assess the appropriateness of an individual's participation given the risks they may be exposed to on Outward Bound courses. In some circumstances, certain medical or psychological conditions may preclude par-

TABLE 2. Foods rich in vitamin $\mathrm{K}$

\section{High vitamin $K$}

- Broccoli

- Brussel sprouts

- Cabbage

- Collard greens

- Green tea

- Kale

- Lettuce (green leaf, romaine)

- Soybeans

- Spinach

\section{Medium vitamin $K$}

- Asparagus

- Green beans

- Lettuce (red leaf)

- Blackberries/blueberries

- Grapes

- Kiwi

- Cashews

- Ensure/Boost nutritional supplements

- Vegetable juice 
TABLE 3. Sports believed to pose a danger of bodily collision

- American football

- Auto racing

- Baseball/softball

- Basketball

- Bobsledding

- Boxing

- Cycling

- Diving

- Downhill skiing

- Equestrian

- Field hockey

- Figure skating

- Gymnastics

- Ice hockey

- Lacrosse

- Luge

- Martial arts

- Motorcycling

- Rock climbing ${ }^{a}$

- Rodeoing

- Rugby

- Skateboarding

- Snowboarding

- Soccer

- Speedskating

- Surfing

- Triathlon

- Water skiing

- Weight-lifting

- Windsurfing

- Wrestling

a "Sport climbing" is categorized by the 36th Bethesda Conference, Eligibility Recommendations for Competitive Athletes with Cardiovascular Abnormalities as a sport at low risk for bodily collision. The European Society of Cardiology classifies "rock climbing" as a sport posing a significant risk of bodily collision. Neither defines their terms sufficiently to know the intended difference (if any) between "sport climbing" and "rock climbing." Our recommendation is that all types of climbing (sport, rock, mountaineering, deep water soloing, gym, and other types) be included in Table 3 .

Table 3 data were derived from Mitchell et al. ${ }^{20}$ and Pelliccia et al. ${ }^{21}$

ticipation. The NCOBS relies heavily on the Outward Bound Screening Manual, developed in collaboration with Wilderness Medical Associates (WMA). In addition, The NCOBS uses "Outward Bound Standards for Participant Screening" to address a number of conditions not addressed in the Screening Manual (such as anticoagulated patients). This document, updated in March 2009, states that students taking warfarin will only be considered for front country courses and will not be accepted for backcountry courses. In the NCOBS nomenclature, "front country" refers to program areas that are within 1 hour of definitive (hospital) medical care.
"Backcountry" refers to those program areas from which a student could not be transported to definitive (hospital) medical care within 1 hour. The Division Safety Director, after appropriate medical consultation, may grant individual exceptions. Typically this consultation includes the patient's physician and the NCOBS's physician medical advisor.

\section{Literature Review and Discussion of Iatrogenic Anticoagulation and Wilderness Activities}

There is a paucity of information in the current medical literature regarding recommendations of physical activity for individuals taking anticoagulants. Micromedex 2.0 advises patients on warfarin to "avoid situations/activities in which cuts, bruising, or injury is likely to occur." "17 Other sources simply make the recommendation of "no contact sports" without specification of what sports were considered to be contact sports. ${ }^{18,19}$ The 36 th Bethesda Conference Eligibility Recommendations for Competitive Athletes with Cardiovascular Abnormalities $^{20}$ (hereafter referred to as the 36th Bethesda Conference) and the European Society of Cardiology ${ }^{21}$ describe a classification of sports (static and dynamic), with a subset of sports that place the patient at risk for danger of a bodily collision (Table 3 ). Bodily collision was defined

TABLE 4. Outdoor sports believed by existing classification systems not to pose a danger of bodily collision

- Archery

- Canoeing/kayaking

- Cricket

- Cross-country skiing

- Decathlon

- Field events (jumping, throwing)

- Golf

- Orienteering

- Race walking

- Riflery

- Rowing

- Running

- Sailing

- Sport climbing ${ }^{a}$

- Swimming

- Tennis

- Volleyball

a "Sport climbing" is categorized by the 36th Bethesda Conference, Eligibility Recommendations for Competitive Athletes with Cardiovascular Abnormalities as a sport at low risk for bodily collision. The European Society of Cardiology classifies "rock climbing" as a sport posing a significant risk of bodily collision. Neither defines their terms sufficiently to know the intended difference (if any) between "sport climbing" and "rock climbing."

Table 4 data were derived from Mitchell et al. ${ }^{20}$ and Pelliccia et al. ${ }^{21}$ 
as a collision caused by a hard impact with another individual, or with an object, a projectile, or the ground. The recommendation for athletes undergoing anticoagulation therapy was to avoid sports that would place the individual at risk for bodily contact or collision. Outdoor sports listed in Table 4 were deemed not to place an individual at risk of bodily collision. However, there appears to be no clinical data for these recommendations, and many anticoagulant experts would not agree with some of the groupings.

Interestingly, the 36th Bethesda Conference classified "sport climbing" as an activity not placing an individual at risk of bodily collision. They did not specifically define sport climbing, but it is typically thought of as a relatively short rock-climbing route using fixed anchors. The European Society of Cardiology classifies "rock climbing" as a sport posing a significant risk of bodily collision. They also did not specifically define rock climbing, so it is impossible to know whether they believe they are looking at the same sport or different forms of climbing. Both groups recognize the limitations of such broad classifications, and the potential for some of these activities to pose less of a risk of bodily collision based on the degree and extent of participation.

There were no citations found that specifically addressed anticoagulation in the wilderness setting. There is abundant literature concerning patients on warfarin involved in trauma. Surprisingly, for injuries not involving the head there is no difference in complications. ${ }^{22}$ Historically, the question of whether anticoagulation increases the risk of complication or death in head-injured patients has been a controversial one, with some initial studies and discussions demonstrating no increased risk, but subsequent studies demonstrating substantially increased risk. ${ }^{22-43}$ However, the most recent publications and data convincingly argue that anticoagulation does significantly increase the risk of intracranial hemorrhage (ICH) in head-injured patients. ${ }^{44}$ One recent meta-analysis of the aggregated data from 8 studies quantifies the risk for computed tomography (CT)-confirmed ICH in anticoagulated patients at 1 in $8 .{ }^{45}$

A significant component to the controversy appears to be that risk may vary in different populations, and increases with advanced age, more significant trauma, or degree of anticoagulation (increased risk with increased INR). ${ }^{32}$ So although there is now broad consensus that anticoagulation increases risk of ICH in all anticoagulated patients, it is less clear what the data would say if extremes of INR and age (average age in the studies being discussed was 60s to 90s) were excluded. This makes it difficult to extrapolate the data available in the medical literature to the specific population of anticoagulated patients most likely to be involved in higher risk activities identified in Table 3, such as a young patient with stable INR involved in rock climbing or snowboarding.

One potential criticism of these data is that, in nearly all cases, it focuses on minor head injury and the end point of interest most often is whether patients who appear to be at low risk should undergo CT scanning. However, this does not appear to be a significant limitation for extrapolation to a wilderness environment. Most certainly a major head injury would be more likely to prompt evacuation in any patient, and the data suggest anticoagulation would be an even more significant prompt to evacuate individuals with significant head injury. However, the criticism might be more relevant for medical screening: because these studies often exclude major head injury, the risk for anticoagulated patients with any type of head injury (minor or major) to have an ICH is almost certainly higher, and thus should prompt even more conservative screening practices.

Another issue concerning wilderness activities for anticoagulated participants is the potential risk to the group or rescuers. Any increased possibility that an anticoagulated participant will require evacuation or increased interventions must be considered in the overall risk profile of that activity. For example, this increased group risk or liability may be minimal for an activity that is an hour away from a major trauma center, versus very significant for a summit attempt on Mount Everest.

The role of other antiplatelet agents, such clopidogrel and aspirin, is less well-studied but worth considering. The National Emergency X-Ray Utilization Study (NEXUS)-II group found the same rate of ICH (1 in 8) in patients suspected to be coagulopathic from any source, including non-warfarin drugs. ${ }^{46}$ More specifically, multiple small studies have suggested clopidogrel use increases risk of $\mathrm{ICH} .{ }^{31,34,47,48} \mathrm{~A}$ more recent and more comprehensive study by the CREST (Clinical Research in Emergency Services and Treatment) Network demonstrates that rates of ICH are, in fact, significantly higher in clopidogrel users (12\% prevalence) than in warfarin users (5\% prevalence). ${ }^{49}$ However, it also showed the risk of delayed ICH (not apparent immediately, even on head CT, but appearing within 14 days) in clopidogrel users was nonexistent, vs a $0.6 \%$ rate prevalence of delayed ICH in warfarin users. ${ }^{49}$ Put into the context of wilderness patients, this suggests that clopidogrel users with head injury should be treated with even more urgency in the immediate postinjury phase than warfarin users, and that for screening purposes clopidogrel users may actually be at higher risk than warfarin users-or at least have equivalent risk. It also suggests that, if evacuation (and CT study) was delayed as a result of wilderness circumstances, the absence of clinical deterioration should be encouraging in clopidogrel users, but may not 
rule out a delayed hemorrhage in a warfarin user. A great concern to wilderness medicine practitioners forced into a "watch-and-see" strategy should be that, in the CREST Network study, $11 \%$ of warfarin users and $18 \%$ of clopidogrel users who had immediate ICH had no loss of consciousness, a normal mental status, and no physical evidence of trauma above the clavicles. ${ }^{49}$ (Immediate $\mathrm{ICH}$ is defined as ICH visible on the first $\mathrm{CT}$ done.) More than $60 \%$ of these patients, regardless of anticoagulant used, had a normal mental status as reflected by Glasgow Coma Score measurement. ${ }^{49}$ This is further argument for avoiding "wait-and-see" strategies when possible based on the CREST Network evidence.

The most salient fact may be that most current clinical guidelines recommend head CT for all anticoagulated patients with head trauma. ${ }^{32,50-52}$ This fact (along with the absence of any actual studies of anticoagulated patients with head injuries in the wilderness indicating they be treated otherwise based on environmental context) suggests that the medicolegal and standard-of-care expectation for these patients would be that they should be taken to a facility for head CT. For those activities in which timely evacuation to a CT-equipped facility is possible, those guidelines imply that anticoagulated patients would increase the risk of trip disruption, the need for rescue, and the potential for evacuation complications. For those activities in which timely evacuation to a CT-equipped facility is not possible, those guidelines have implications for whether or not those individuals should even be participating in the activity.

It is also important to consider the context of these data. Can the studies done on older patients even be applied to younger patients who have head injury? Most of the studies reviewed are intended for clinicians in a healthcare facility who must decide whether or not to obtain a head CT in older anticoagulated patients. This may be the identical question for most wilderness medicine practitioners (with evacuation, or screening an anticoagulated patient onto a trip, as the functional end point analog for head CT). However, for some austere or wilderness medicine practitioners this may not be the right question. The group that these data do not help includes practitioners working in fixed, remote areas (with no reasonable access to CT ever), practitioners working on expeditions for which evacuation is not feasible, or practitioners for mission-imperative activities (rescue work, military operations, etc). In these cases, the clinical significance of the ICH may be as or more important than its presence. In other words, once the decision is passed as to whether or not the anticoagulated patient will be in the operational environment (or that a patient who lives permanently in this environment will be anticoagulated), the presence of an ICH may be irrel- evant if it has no clinical consequences or has no interventional remediation. Although recent aggregate data suggest that delayed hemorrhage may occur in about 1 of every 25 anticoagulated patients even after a normal initial head CT, in all the patients studied in aggregate $(n=224)$ only a single patient required craniotomy. ${ }^{39}$ The question of whether identification would improve outcome is extremely important. It is perhaps more relevant in the remote, austere environment than in the traditional operational environment, and is not necessarily addressed by any of these studies, whose end point is generally simple identification of $\mathrm{ICH}$, not subsequent intervention and outcome. This has important consequences for evacuation decisions and for the relative wilderness significance of the 1 in 8 incidence rate of ICH cited earlier.

\section{Case Resolution}

The Outward Bound physician consulted the literature available and peers in the wilderness medicine community and realized there was little or no pertinent literature available. The NCOBS policy traditionally had banned any anticoagulated individuals from participating in wilderness activities, but this position was now being questioned (in part as a result of this particular case, and the number of people, including increasingly younger and otherwise healthier people, now on lifetime or long-term anticoagulation). More progressive perspectives seem to indicate that type of activity, distance from care, and underlying rationale for anticoagulation might be used to create more individualized criteria for participation, or at least individual review rather than a blanket prohibition.

In this particular case the Outward Bound physician, in consultation with Outward Bound leadership and risk management staff, recommended that the participant be permitted to engage in the hiking portion of the course. Suggestions were made to limit weight to try to avoid trauma in case of a fall. The physician advised that the patient not be permitted to rock climb. In part this was because falling and blunt or suspension trauma- even extremely minor trauma-would not be routinely expected to be part of a novice hiking experience, but would be routinely expected as part of a novice top-rope experience.

The Outward Bound physician also alerted the Burke County Special Operations Team, responsible for wilderness medical care in the National Forest and Wilderness Area, and MedCenter Air, the nearest air medical unit, of the days of the activity and the relevant history of the student. Both organizations expressed appreciation for this advance notification.

The student did participate in the hiking portion of the program, but was an observer only for the rock-climbing 
portion. There were no medical incidents involving this patient during the course.

\section{Recommendations}

For a patient who plans to spend considerable time in outdoor activities there are several options for anticoagulation.

The evaluation of an anticoagulated individual wishing to engage in a wilderness or outdoor activity begins with determining the initial indication for anticoagulation with expected duration of therapy. Certainly a patient with a mechanical valve requires long-term anticoagulation, but a young woman with a birth control-related thrombotic event 8 months ago may be able to stop anticoagulation.

If it is determined that a patient needs to remain anticoagulated, there are several options from which to choose. For a relatively short trip, in the patient with an INR established as therapeutically stable, the patient can simply stay on his or her routine dose of warfarin. This also assumes no dramatic changes in diet or activity, which is not possible with some outdoor adventures. So for a camping trip up to a week the patient would stay on his usual dose, but for longer trips-especially with change in diet or activity level (eg, a long hike for an office worker) - this approach has potential for nontherapeutic INR.

Another option for trips longer than a week is to selfmonitor. Studies have shown that patients who monitor their own INR will be in range more often. ${ }^{53}$ The advantage of this approach is the ability to adjust the warfarin dose over time or if illness strikes. Downsides are the need to travel with the machine and cartridge, and the patient will need an algorithm for dose adjustment.

One could also change to alternative anticoagulants. Low-molecular-weight heparin (LMWH) agents such as enoxaparin (Lovenox) will provide stable dosing for all patients, but this plan requires that the patient must travel with all required equipment and supplies for sterile subcutaneous administration of the medication. There may be hygiene or infection issues regarding frequent routine injections in a wilderness setting. The new antithrombotic agents - apixaban, dabigatran and rivaroxaban — have indications for venous thrombosis and stroke prevention. ${ }^{54}$ These agents offer the advantage of both stable anticoagulation and oral administration. Given the stability of anticoagulation owing to lack of food or drug interactions with both LMWH and the new oral agents, the risk of bleeding or thrombosis should be less. In addition, the new oral agents have been shown to have a lesser risk of ICH than warfarin. A downside is that they have been tested only for limited indications and cannot be used for patients with cardiac valves. However, for long trips or situations in which there will be inconsistency of diet, changing to one of these alternative anticoagulants might be an attractive option.
If the patient is planning to start any new medications for the trip, one strategy is to start the medication a week or more in advance to ensure there are no drug interactions. The patient may also want to carry a list of drugs that interact with warfarin (Table 1), as medical providers may not have access to this information in an austere setting.

Ultimately, binary recommendations (yes or no) need to be made for 2 actions: preactivity medical screening of anticoagulated patients for wilderness activities, and treating anticoagulated patients involved in wilderness activities. The following are our recommendations for these actions:

\section{PREACTIVITY MEDICAL SCREENING}

A. Patients should not routinely participate in activities placing them at risk of bodily collision while anticoagulated (Table 3).

1. Considerations for exceptions:

a. Duration of therapy

i. Limited-defer until duration of therapy is complete

ii. Lifetime-consider risk to individual and risk to others

b. Risk to individual—considerations

i. Expertise of individual

ii. Stability of INR if on warfarin

iii. Alternatives to warfarin vs risk of substandard care for indications of anticoagulation

iv. Duration of event

v. Distance from definitive care and degree of evacuation difficulty

vi. Risk of significant injury

c. Risks to others-considerations

i. Distance from definitive care and degree of evacuation difficulty

ii. Expertise of others in evaluation and treatment of ICH and potential ICH or other hemorrhagic complications while in the specified setting of the event

iii. Willingness and ability to fully support the anticoagulated individual in the event of injury or unfavorable circumstances, including the willingness to abandon the goal of the trip to care for the anticoagulated individual. (This may specifically relate to a private vs commercial venture).

B. Anticoagulated patients pursuing programs involving activities that do not place them at risk of bodily collision (Table 4) should be screened on or off based on relative risk-benefit analysis. This analysis should be performed collaboratively, to include the patient, his or her personal physician, the program staff, and 
ideally the program medical advisor. The risk portion of this analysis should include the expertise of the patient, the character of the environment including distance from medical care, and, in the case of warfarin, stability of INR.

C. In both cases (A and B above), available evidence suggests that clopidogrel is at least as dangerous as warfarin in terms of prevalence of ICH after head injury. This should be considered as a factor in preactivity medical screening.

\section{TREATMENT AND EVACUATION}

A. We recommend that patients anticoagulated with clopidogrel or warfarin involved in traumatic incidents not involving head injury or significant hemorrhage be treated similarly to nonanticoagulated patients, with only slightly heightened conservatism based on increased theoretical risk for bleeding sequelae. However, there does not appear to be compelling evidence that these patients need to be automatically evacuated or treated as having objectively verifiable increased risk of bleeding sequelae.

B. We recommend that every effort be made to prevent head injury in anticoagulated patients (warfarin or clopidogrel) during wilderness activities. This includes helmet use and activity modification whenever possible and reasonable.

C. We recommend any patient anticoagulated who sustains a head injury and is taking clopidogrel or warfarin be evacuated, when feasible, regardless of clinical symptoms. Note that clopidogrel patients may have an increased risk of immediate ICH versus warfarin patients, but warfarin patients have an increased risk of delayed ICH (not initially apparent radiographically, but appearing within 14 days), which does not appear to be a concern with clopidogrel. Note however that there appears to be a low rate of need for surgical intervention in these cases of delayed $\mathrm{ICH}$.

\section{Conclusions}

There are inadequate resources in the literature for consultant wilderness physicians to advise organizations on risk management or to follow evidence-based practices in treating coagulopathic patients in wilderness settings. This case study and literature review begins to address that paucity of resources, and includes recommendations based on the research currently available. More research is required to fully understand the role and risks of anticoagulation in the wilderness.

\section{References}

1. Anticoagulation; Coagulopathy. The Merriam-Webster Dictionary. Available at http://www.merriam-webster. com/medical/anticoagulation; http://www.merriamwebster.com/medical/coagulopathy. Accessed June 14, 2012.

2. Galanis T, Thomson L, Palladino M, Merli GJ. New oral anticoagulants. J Thromb Thrombolysis. 2011;31: 310-320.

3. Thinking outside the box: 50 years of Outward Bound. The Private Journey Magazine. Spring 2012. Available at http://www.ncobs.org/lib/file/manager/TPJ_Spring_single_ OutwardBound.pdf. Accessed June 14, 2012.

4. Ansell J, Hirsh J, Hylek E, Jacobson A, Crowther M, Palareti G; American College of Chest Physicians. Pharmacology and management of the vitamin $\mathrm{K}$ antagonists: American College of Chest Physicians Evidence-Based Clinical Practice Guidelines (8th Edition). Chest. 2008; 133(6 suppl):160S-198S.

5. Schulman S, Parpia S, Stewart C, Rudd-Scott L, Julian JA, Levine M. Warfarin dose assessment every 4 weeks versus every 12 weeks in patients with stable international normalized ratios: a randomized trial. Ann Intern Med. 2011;155:653-659.

6. Booth SL, Centurelli MA. Vitamin K: a practical guide to the dietary management of patients on warfarin. Nutr Rev. 1999;57(9 Pt 1):288-296.

7. Sconce E, Khan T, Mason J, Noble F, Wynne H, Kamali F. Patients with unstable control have a poorer dietary intake of vitamin $\mathrm{K}$ compared to patients with stable control of anticoagulation. Thromb Haemost. 2005;93:872-875.

8. Van Patot MC, Hill AE, Dingmann C, et al. Risk of impaired coagulation in warfarin patients ascending to altitude (>2400 m). High Alt Med Biol. 2006;7:39-46.

9. Hansen ML, Sørensen R, Clausen MT, et al. Risk of bleeding with single, dual, or triple therapy with warfarin, aspirin, and clopidogrel in patients with atrial fibrillation. Arch Intern Med. 2010;170:1433-1441.

10. DeLoughery TG. Critical care clotting catastrophies. Crit Care Clin. 2005;21:531-562.

11. Nutescu E, Chuatrisorn I, Hellenbart E. Drug and dietary interactions of warfarin and novel oral anticoagulants: an update. J Thromb Thrombolysis. 2011;31:326-343.

12. White PJ. Patient factors that influence warfarin dose response. J Pharm Pract. 2010;23:194-204.

13. Bungard TJ, Yakiwchuk E, Foisy M, Brocklebank C. Drug interactions involving warfarin: practice tool and practical management tips. Can Pharm J. 2011;144:21-52.

14. Holbrook AM, Pereira JA, Labiris R, et al. Systematic overview of warfarin and its drug and food interactions. Arch Intern Med. 2005;165:1095-1106.

15. Weitz JI, Eikelboom JW, Samama MM; American College of Chest Physicians. New antithrombotic drugs: Antithrombotic Therapy and Prevention of Thrombosis, 9th ed: American College of Chest Physicians Evidence-Based Clinical Practice Guidelines. Chest. 2012:141(2 suppl):e120S-151S. 
16. Gennari L, Deguzman FR, Lakhotia S, et al. Recombinant antidote for reversal of anticoagulation by factor Xa inhibitors. Blood 2008;112(abstract).

17. Warfarin Sodium, DrugPoint Summary Clinical Teaching. Micromedex, copyright 1974-2011 Thomson Reuters. Last modified: November 3, 2011.

18. Mellwig KP, van Buuren F, Gohlke-Baerwolf C, Bjørnstad $\mathrm{HH}$. Recommendations for the management of individuals with acquired valvular heart diseases who are involved in leisure-time physical activities or competitive sports. Eur J Cardovasc Prev Rehabil. 2008;15:95-103.

19. Depenbrock PJ. Thromboembolic disorders: guidance for return-to-play. Curr Sports Med Rep. 2011;10:78-83.

20. Mitchell JH, Haskell W, Snell P, Van Camp SP. Task Force 8: classification of sports (part of the 36th Bethesda Conference, Eligibility Recommendations for Competitive Athletes with Cardiovascular Abnormalities). J Am Coll Cardiol. 2005;45:1364-1367.

21. Pelliccia A, Fagard R, Bjørnstad HH, et al. Recommendations for competitive sports participation in athletes with cardiovascular disease: a consensus document from the Study Group of Sports Cardiology of the Working Group of Cardiac Rehabilitation and Exercise Physiology and the Working Group of Myocardial and Pericardial Diseases of the European Society of Cardiology. Eur Heart J. 2005; 26:1422-1445.

22. Mina AA, Bair HA, Howells GA, Bendick PJ. Complications of preinjury warfarin use in the trauma patient. J Trauma. 2003;54:842-847.

23. Ott MM, Eriksson E, Vanderkolk W, Christianson D, Davis A, Scholten D. Antiplatelet and anticoagulation therapies do not increase mortality in the absence of traumatic brain injury. J Trauma. 2010;68:560-563.

24. Ahmed N, Bialowas C, Kuo YH, Zawodniak L. Impact of preinjury anticoagulation in patients with traumatic brain injury. South Med J. 2009;102:476-480.

25. Franko J, Kish KJ, O'Connell BG, Subramanian S, Yuschak JV. Advanced age and preinjury warfarin anticoagulation increase the risk of mortality after head trauma. J Trauma. 2006;61:107-110.

26. McMillian WD, Rogers FB. Management of prehospital antiplatelet and anticoagulant therapy in traumatic head injury: a review. J Trauma. 2009;66:942-950.

27. Wojcik R, Cipolle MD, Seislove E, Wasser TE, Pasquale MD. Preinjury warfarin does not impact outcome in trauma patients. J Trauma 2001;51:1147-1152.

28. Garra G, Nashed AH, Capobianco L. Minor head trauma in anticoagulated patients. Acad Emerg Med. 1999;6:121-124.

29. Ibañez J, Arikan F, Pedraza S, et al. Reliability of clinical guidelines in the detection of patients at risk following mild head injury: results of a prospective study. J Neurosurg. 2004;100:825-834.

30. Fabbri A, Servadei F, Marchesini G, et al. Clinical performance of NICE recommendations versus NCWFNS proposal in patients with mild head injury. J Neurotrauma. 2005;22:1419-1427.
31. Cohen DB, Rinker C, Wilberger JE. Traumatic brain injury in anticoagulated patients. J Trauma. 2006;60:553-557.

32. Menditto VG, Lucci M, Polonara S, Pomponio G, Gabrielli A. Management of minor head injury in patients receiving oral anticoagulation therapy: a prospective study of a 24-hour observation protocol. Ann Emerg Med. 2012;59:451-455.

33. Volans AP. The risks of minor head injury in the warfarinised patient. J Accid Emerg Med. 1998;15:159-161.

34. Itshayek E, Rosenthal G, Fraifeld S, Perez-Sanchez X, Cohen JE, Spektor S. Delayed posttraumatic acute subdural hematoma in elderly patients on anticoagulation. Neurosurgery. 2006;58:E851-E856.

35. Li J, Brown J, Levine M. Mild head injury, anticoagulants, and risk of intracranial injury. Lancet. 2001;357;771-772.

36. Claudia C, Claudia R, Agostino O, Simone M, Stefano G. Minor head injury in warfarinized patients: indicators of risk for intracranial hemorrhage. J Trauma. 2011;70:906-909.

37. Franko J, Kish KJ, O'Connell BG, Subramanian S, Yuschak JV. Advanced age and preinjury warfarin anticoagulation increase the risk of mortality after head trauma. J Trauma. 2006;61:107-110.

38. Gittleman AM, Ortiz AO, Keating DP, Katz DS. Indications for $\mathrm{CT}$ in patients receiving anticoagulation after head trauma. AJNR Am J Neuroradiol. 2005;26:603-606.

39. Major J, Reed MJ. A retrospective review of patients with head injury with coexistent anticoagulant and antiplatelet use admitted from a UK emergency department. Emerg Med J. 2009;26:871-876.

40. Servadei F, Teasdale G, Merry G; Neurotraumatology Committee of the World Federation of Neurosurgical Societies. Defining acute mild head injury in adults: a proposal based on prognostic factors, diagnosis and management. J Neurotrauma. 2001;18:657-664.

41. Leiblich A Mason S. Emergency management of minor head injury in anticoagulated patients. Emerg Med J. 2011; 28:115-118.

42. Lavoie A, Ratte S, Clas D, et al. Preinjury warfarin use among elderly patients with closed head injuries in a trauma center. J Trauma. 2004;56:802-807.

43. Karni A, Holtzman R, Bass T, et al. Traumatic head injury in the anticoagulated elderly patient: a lethal combination. Am Surg. 2001;67:1098-1100.

44. Li J. Admit all anticoagulated head-injured patients? A million dollars versus your dime. You make the call. Ann Emerg Med. 2012;59:457-459.

45. Li J. Validation of the dime. Ann Emerg Med. 2012;59: 469-470.

46. Medzon R, Bracken M, Rathlev NK, et al. Clinically suspected coagulopathy in blunt head trauma. J Emerg Med. 2010;39:399-405.

47. Jones K, Sharp C, Mangram AJ, Dunn EL. The effects of preinjury clopidogrel use on older trauma patients with head injuries. Am J Surg. 2006;192:743-745.

48. Brewer ES, Reznikov B, Liberman F, et al. Incidence and predictors of intracranial hemorrhage after minor head trauma in patients taking anticoagulant and antiplatelet medication. J Trauma. 2011;70:E1-5. 
49. Nishijima DK, Offerman SR, Ballard DW, et al. Immediate and delayed traumatic intracranial hemorrhage in patients with head trauma and preinjury warfarin or clopidogrel use. Ann Emerg Med. 2012;59:460-468.

50. Jagoda AS, Bazarian JJ, Bruns JJ Jr, et al; American College of Emergency Physicians; Centers for Disease Control and Prevention. Clinical policy: neuroimaging and decisionmaking in adult mild traumatic brain injury in the acute setting. Ann Emerg Med. 2008;52;714-748.

51. New Zealand Guidelines Group. Traumatic Brain Injury: Diagnosis, Acute Management and Rehabilita- tion. Wellington, New Zealand: New Zealand Guidelines Group; 2006.

52. National Collaborating Centre for Acute Care. Head Injury: Triage, Assessment, Investigation and Early Management of Head Injury in Infants, Children and Adults. London, UK: National Institute for Health and Clinical Excellence; 2007.

53. Matchar DB, Jacobson A, Dolor R, et al. Effect of home testing of international normalized ratio on clinical events. N Engl J Med. 2010;363:1608-1620.

54. DeLoughery TG. Practical aspects of the oral new anticoagulants. Am J Hematol. 2011;86:586-590. 